Article

\title{
Effect of Graphene Coating on the Heat Transfer Performance of a Composite Anti-/Deicing Component
}

\author{
Long Chen ${ }^{(D)}$, Yidu Zhang and Qiong Wu * \\ State Key Laboratory of Virtual Reality Technology, School of Mechanical Engineering and Automation, \\ Beihang University, Beijing 100191, China; dragonchen@buaa.edu.cn (L.C.); ydzhang@buaa.edu.cn (Y.Z.) \\ * Correspondence: wuqiong@buaa.edu.cn; Tel.: +86-10-8231-7756
}

Received: 15 August 2017; Accepted: 25 September 2017; Published: 28 September 2017

\begin{abstract}
The thermal conductivity of a graphene coating for anti-/deicing is rarely studied. This paper presents an improved anti-/deicing efficiency method for composite material anti-/deicing by using the heat-transfer characteristic of a graphene coating. An anti-/deicing experiment was conducted using the centrifugal force generated by a helicopter rotor. Results showed that the graphene coating can accelerate the internal heat transfer of the composite material, thereby improving the anti-icing and deicing efficiency of the helicopter rotor. The spraying process parameters, such as coating thickness and spraying pressure, were also studied. Results showed that reducing coating thickness and increasing spraying pressure are beneficial in preparing a graphene coating with high thermal conductivity. This study provides an experimental reference for the application of a graphene coating in anti-/deicing.
\end{abstract}

Keywords: graphene coating; anti-/deicing; thermal conductivity; composite material

\section{Introduction}

When a helicopter is flying at high altitude or in a cold zone, super-cooled water droplets in the air will impact on the surface of the rotor, icing rapidly. As the rotor is the main device providing lift for the helicopter, icing on the surface will undermine its aerodynamic shape, severely reducing its aerodynamic characteristics and threatening helicopter flight safety [1-3]. Existing ice control technology for helicopters is classified in terms of active anti-/deicing and passive anti-/deicing. Surface coatings with anti-/deicing properties are being explored as passive methods to prevent water droplets from icing on the anti-icing surface or to reduce the adhesion force between the ice and deicing surface. A series of materials, such as carbon-based material [4-6], liquid-infused material [7-9], and nanocomposites [10-12], exhibit anti-icing functions. Most of these materials are suitable for anti-icing in a limited number of icing conditions, while the effect is not ideal under moderate icing conditions. Active anti-/deicing technology relies mainly upon on-board energy [13-16] or mechanical energy [17-19]. This kind of anti-/deicing technology displays high deicing efficiency, but the energy consumption is high as well. Therefore, existing technology cannot satisfy the requirements of anti-/deicing completely and effectively, and a synthesis that combines surface coating with passive deicing should be developed to achieve the dual effect of anti-icing and deicing.

As a hexagonal carbon reticular linkage material, graphene displays excellent electrical $[20,21]$ and thermal conductivities [22,23]. Research on graphene applications has been conducted by many scholars. In terms of the conductive properties of graphene, Hong et al. [24] and Choi et al. [25] fabricated a graphene film with low surface resistance and high optical transmittance. The film is superior to the traditional transparent heater based on tin cobalt oxide and is widely used in automobile and deicing defogging systems. In terms of energy storage devices, self-assembled graphene/carbon 
nanotube hybridfilms for supercapacitors were fabricated by Dai et al. [26] and Ruoff et al. [27]. These films exhibit outstanding high-scanning speed and capacitance and are used in the manufacture of batteries and micro-computers. In addition, graphene is used as conductive ink for application in the printing field. Magdasi et al. [28] improved the inkjet printing of ink formulation by adding graphene sheet and metal nanoparticles to printing ink suitable for the printing of electronic products. Moreover, Hersam et al. [29] performed fast post-processing of printed electronics by intense pulsed light annealing graphene on different substrates, which perfroms well in printing and electronic products. Graphene is also widely used in other applications. Lu et al. [30] developed a new type of graphene-coated nickel electrode. Studies have shown that graphene coating has a significant effect on electrode corrosion resistance. In the research of Wan [31], an ultrafast atmospheric-pressure plasma jet (APPJ) processed Pt-decorated reduced graphene oxides (rGOs) that were used as counter-electrodes in dye-sensitized solar cells (DSSCs). The reduced graphene oxides improve the efficiency of the dye-sensitized cells.

However, the application of graphene in the anti-/deicing field is rarely studied. Currently, graphene is used in the field of anti-icing and deicing by utilizing its high conductivity property. Graphene conducts electricity, and by applying a voltage at the poles of the graphene coating, the current causes Joule heat to melt the ice. Thus, the electrical energy is converted into Joule heat to remove ice from a static helicopter rotor blade [32,33]. Subsequently, a graphene coating with anti-icing and deicing ability was developed by applying the lubricant [34].

The heat-transfer analysis of engineering applications using numerical methods have been proposed by many scholars. Ellahi et al. [35] created a model to discuss diverse issues related to heat transfer. Rashidi et al. [36] proposed a two-way coupling of a discrete phase model in order to track the discrete nature of aluminum oxide particles in an obstructed duct with two side-by-side obstacles. Shirvan et al. [37] carried out a 2-D numerical simulation and sensitivity analysis on turbulent heat transfer and heat exchanger effectiveness enhancement, and a numerical study that investigated natural convection along with surface radiation heat transfer in an inclined porous solar cavity [38,39]. Bhatti et al. [40] investigated the electromagnetohydrodynamic (EMHD) flow with heat transfer on third-grade fluid containing small particles. These references provide numerical methods for studying heat transfer. However, due to the complexity of the cross-scale heat transfer of micro materials, there is little literature on the analysis of the thermal conductivity of graphene materials using numerical methods.

Based on the existing research results, this paper presents a method that takes advantage of the heat-transfer characteristics of graphene to combine it with the traditional electric heating method for helicopter rotor anti-/deicing. A method combining a graphene coating with the anti-/deicing component is proposed to enhance the anti-/deicing efficiency of the rotor. A deicing experiment with graphene coating was conducted in an environment at $-15^{\circ} \mathrm{C}$. Results showed that the deicing effect is improved with a graphene coating.

\section{Experimental Procedure}

\subsection{Materials}

Liquid graphene was purchased from Jiangsu Tanfeng Graphene Technology Co. Ltd. (Suzhou, China), and was used without any further treatment. Liquid graphene is a kind of nanomaterial that distributes surface heat in the form of radiation and enhances heat conduction. This material also shows tolerance at a high temperature range of $20-180^{\circ} \mathrm{C}$. The emissivity of the coating to the atmosphere or the inner space of the object is approximately $\varepsilon>0.92$, which can accelerate heat conduction and improve the heat exchange rate. 


\subsection{Characterization}

The sample structures were investigated by scanning electron microscopy (SEM, FEI Quanta 250 FEG, FEI, Hillsboro, OR, USA) and transmission electron micrographs (TEM, FEI, Hillsboro, OR, USA). Prior to SEM analysis, a thin Au layer was deposited on the specimens by sputtering. The morphologies and sizes of graphene were observed by an SEM operated at an acceleration voltage of $10 \mathrm{kV}$ and standard current. The magnification was 5000. The TEM image was provided by the Jiangsu Tanfeng Graphene Technology Co. Ltd.

\subsection{Preparation of Graphene Coating Samples}

The graphene coating samples were prepared using trial spraying. The thickness of the graphene coating, the pressure of the spray pump, and the caliber of the spray gun were adjusted in the trial spraying. After determining the thickness of the coating, a stainless steel surface $(100 \mathrm{~mm} \times 100 \mathrm{~mm})$ was sprayed. The graphene coating was stirred for $3 \mathrm{~min}$ at ambient temperature before the substrate was painted. During the spraying process, the distance between the nozzle and the surface of the sample was maintained, and the spraying pressure was controlled to avoid spraying twice. The graphene coating samples were heated in an oven at $80^{\circ} \mathrm{C}$ for $1 \mathrm{~h}$ for curing. In order to study the relationship between spraying pressure and the heat transfer of the graphene coating, the spraying pressure was varied at 20, 40, 60 and 80 psi.

\subsection{Preparation of Anti-/Deicing Component}

The anti-/deicing component was fabricated by using carbon and glass fibers (Figure 1). The insulation layer was made by utilizing six layers of $3 \mathrm{~K}$ prepreg epoxy resin carbon fiber. The heat transfer layer of the anti-/deicing component was made of three layers prepreg epoxy resin. Each layer of material was cemented with adhesive. Electric heating wires were arranged between the insulation heat layer and the heat transfer layer to be cemented and fixed. Eventually, the whole anti-/deicing component was heated in a heating box at $125^{\circ} \mathrm{C}$ for $1 \mathrm{~h}$ for curing.

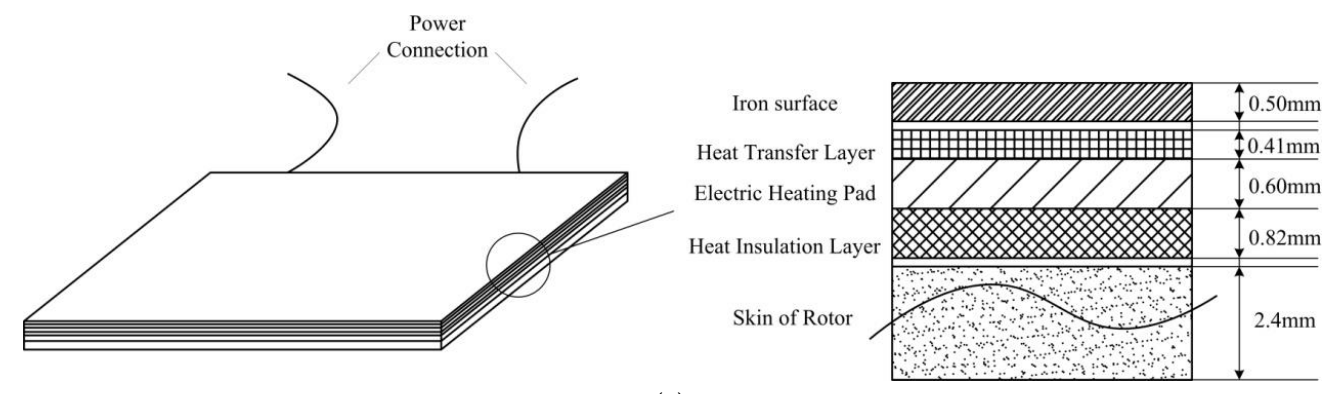

(a)

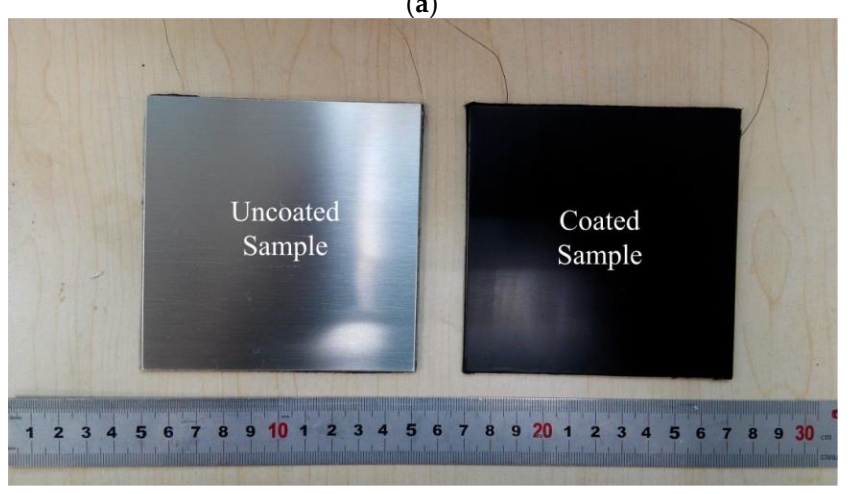

(b)

Figure 1. (a) Schematic of anti-/deicing component without graphene coating; (b) Photograph of uncoated sample and coated sample. 


\subsection{Anti-/Deicing Component Heating and Deicing Experiments}

The anti-/deicing component was heated by applying a DC power device (Figure 2). A constant voltage was applied across electrodes on the two ends of the heating wire in the experiment, and the power was maintained. The deicing experiment was carried out in the refrigeration box, where the temperature was stabilized at $-15{ }^{\circ} \mathrm{C}$ by the automatic constant temperature refrigeration system. The icing thickness was $15 \mathrm{~mm}$ according to medium icing conditions. The setting of experimental conditions was determined according to the general trial standard, and the ice thickness was chosen as the average value of the medium icing thickness range. Super-cooled water was dropped onto the surfaces of the test sample to form a predetermined icing thickness.

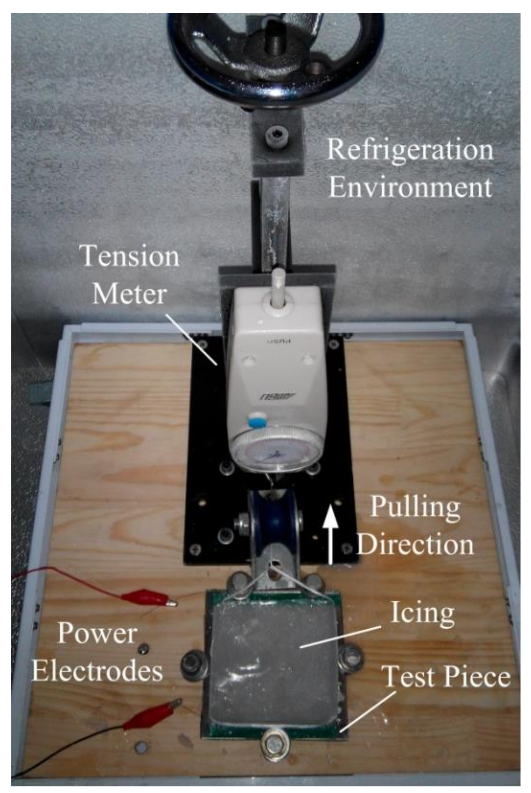

Figure 2. Photograph of deicing experiment device.

In order to achieve an equivalent centrifugal force generated by high-speed rotation of the rotor, a tensionmeter was used to provide a tension equal to the centrifugal force. The measuring range of the tensionmeter was $0-500 \mathrm{~N}$, and the accuracy was $0.5 \mathrm{~N}$. The tension $F$ was calculated by the following equation:

$$
F=m_{\text {ice }} \frac{v^{2}}{r}
$$

where $m_{\text {ice }}$ is the mass of the icing, $v$ is the rotating linear velocity of the icing, and $r$ is the radius of gyration.

In the experiments, the effect of refrigeration temperature fluctuations with measurement error of the thermocouple sensor was likely to cause errors in the data. By averaging the measured value of the experimental results, the error was effectively reduced.

\section{Results and Discussion}

\subsection{Graphene Coating Characterizations}

Figure 3 shows the general TEM and SEM overviews of the graphene coating. Figure 3a illustrates that the graphene possesses a thin translucent sheet structure distribution. In the SEM image, the graphene exhibits a continuous two-dimensional lamellar structure with wavy folds. From the SEM images, we can see that the graphene forms an uneven surface with folds of a certain thickness, and there are obvious wrinkles. With the increase in the graphene layer, the degree of folding becomes smaller. In the image, the color is deeper with thicker graphene layers, and shallow when the 
graphene layer is relatively thin. In addition, the graphene is stable in air at room temperature, and oxidative decomposition only commences above $400{ }^{\circ} \mathrm{C}$ according to thermogravimetric analysis [33]. The material's structural and chemical stability make graphene suitable for deicing applications.

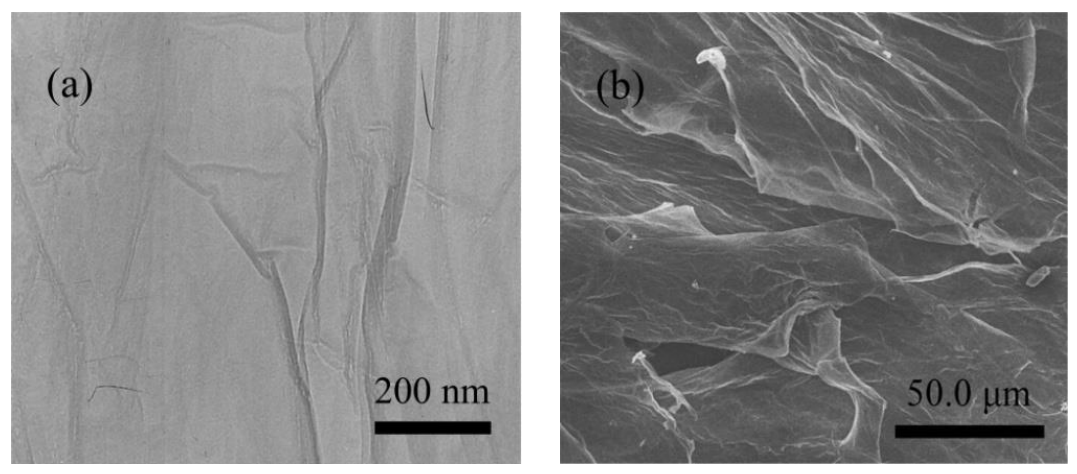

Figure 3. (a) Transmission electron microscopy (TEM), and (b) scanning electron microscopy (SEM) images of the graphene coating.

\subsection{Anti-Icing Property}

To verify the anti-icing effect of the graphene coating, the surface temperature of the test sample with the graphene coating at $-15^{\circ} \mathrm{C}$ was measured using an infrared thermometer with a spot size of $\sim 0.5 \mathrm{~cm}$. In the experiment, the measuring range of the thermometer was between $-50{ }^{\circ} \mathrm{C}$ and $380^{\circ} \mathrm{C}$, and the accuracy was $0.5^{\circ} \mathrm{C}$. The reported accuracy for the thermocouple also takes account of uncertainty due to the data acquisition software. In Figure 3, the tests were conducted under stable cooling. There are limitations to the experimental conditions, because super-cooled water droplets in actual situations do not form easily. Therefore, the anti-icing requirements for a helicopter rotor in reference [41] were adopted in the experiment. The rotor will not freeze when the surface temperature exceeds $2{ }^{\circ} \mathrm{C}$.

In the experiment, the selected electric voltages were 10.9, 11.5, and $12.9 \mathrm{~V}$. The surface temperature of the anti-/deicing component was measured every $50 \mathrm{~s}$. The heating profile of the anti-/deicing component under different heating conditions is shown in Figure 4. Comparison of the temperature curves showed that the surface temperature of the uncoated anti-/deicing component was lower than that of the graphene-coated samples in different heating conditions. With the increase in heating voltage, the surface temperature of the anti-/deicing component also increased. Under the three experimental conditions, the temperatures increased by $1.7^{\circ} \mathrm{C}, 2.2^{\circ} \mathrm{C}$ and $2.8^{\circ} \mathrm{C}$, respectively.

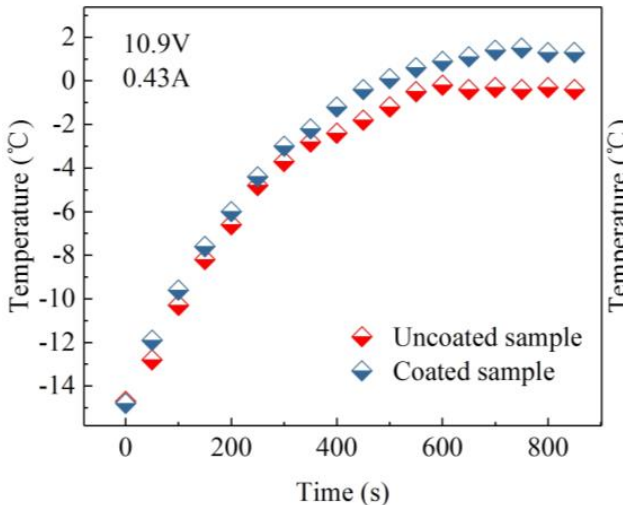

(a)

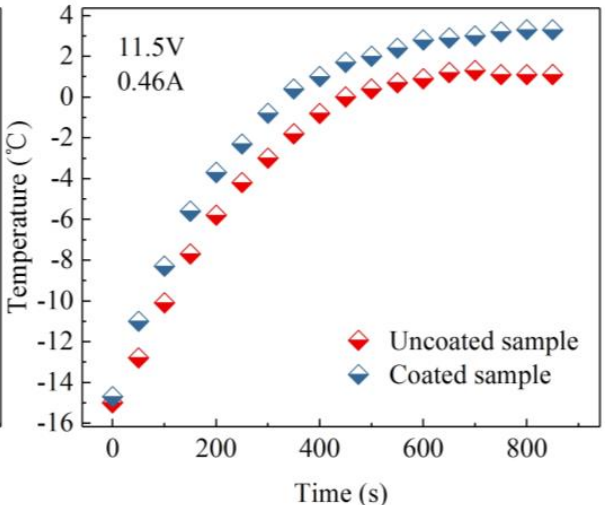

(b)

Figure 4. Cont. 


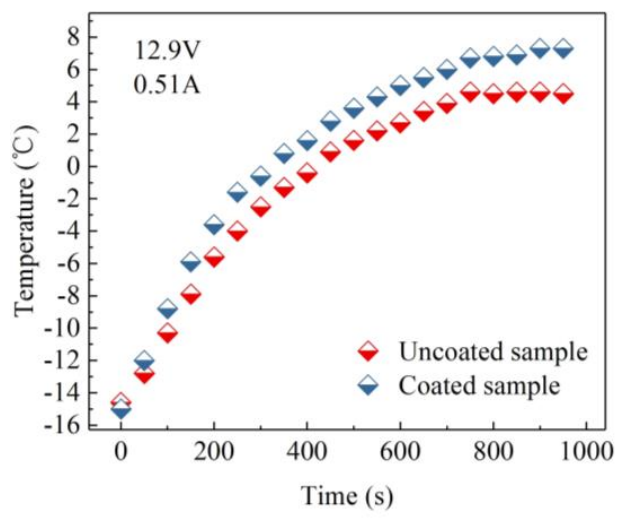

(c)

Figure 4. Temperature profile of the anti-/deicing component at different applied voltages. (a) Applied voltage: $10.9 \mathrm{~V}, 0.43 \mathrm{~A}$; (b) applied voltage: $11.5 \mathrm{~V}, 0.46 \mathrm{~A}$; (c) applied voltage: $12.9 \mathrm{~V}, 0.51 \mathrm{~A}$.

Under the first heating condition, the surface temperature of the uncoated test sample did not reach freezing point, and the coating test sample reached more than $0{ }^{\circ} \mathrm{C}$. Under the second set of heating conditions, the uncoated test sample surface temperature was less than $2{ }^{\circ} \mathrm{C}$, but the surface temperature of the coated test sample satisfied the anti-/deicing requirements. This result indicated that the anti-/deicing component with a graphene coating possesses anti-/deicing capability in this heating condition. In the third set, the surface temperature of uncoated and coated test samples both reached $2{ }^{\circ} \mathrm{C}$, and the heating condition may result in the loss of on-board energy. Furthermore, analysis of the temperature curves evidently showed that the temperature of coated test samples was higher than that of uncoated test samples. With the increase in heating voltage, the surface temperature difference between coated and uncoated samples also increased. The experimental results indicated that the thermal conductivity of the graphene coating is good, and the anti-/deicing performance is ideal at a high temperature. The graphene coating enhances the heat transfer capability of the iron surface, which transfers more heat to the surface of the rotor under the same heating conditions. The results indicate that the graphene coating is helpful in anti-/deicing.

\subsection{Deicing Property}

The deicing experiment of the graphene coating is described in detail in Section 2.4. The heating currents adopted in the deicing experiment were $0.77,1.03$, and $1.18 \mathrm{~A}$, and the corresponding heating voltages were $20.2,24.9$, and $29.9 \mathrm{~V}$, respectively. The experimental tests were conducted with coated and uncoated samples. The centrifugal force calculation method is introduced in Section 2.4. According to Equation (1), a $64 \mathrm{~N}$ centrifugal force was exerted upon the surface icing of the anti-/deicing component. The experiments were carried out in an environment at $-15{ }^{\circ} \mathrm{C}$. The deicing time was recorded by using a stopwatch, and data are shown in Figure 5.

The deicing time of coated and uncoated test samples was compared. Results showed that the deicing time of coated test sample was significantly shorter than that of uncoated test samples, which indicated that graphene coating can significantly improve the deicing efficiency of the anti-/deicing component. The deicing time decreased by $41.64 \%, 57.42 \%$, and $71.74 \%$, respectively. Shorter deicing times considerably reduced the energy consumption of the helicopter.

Analysis demonstrated that with the increase in heating voltage and current, the deicing time of the coated test and uncoated test samples was reduced; this observation implied that the deicing efficiency of the anti-/deicing component was improved. In Section 3.1, the surface temperatures of the coated and uncoated test samples increased with the increasing heating power. The surface temperature increase of the coated test sample was higher than that of uncoated test sample, which indirectly explained the reliability of the experimental results in Figure 5. 


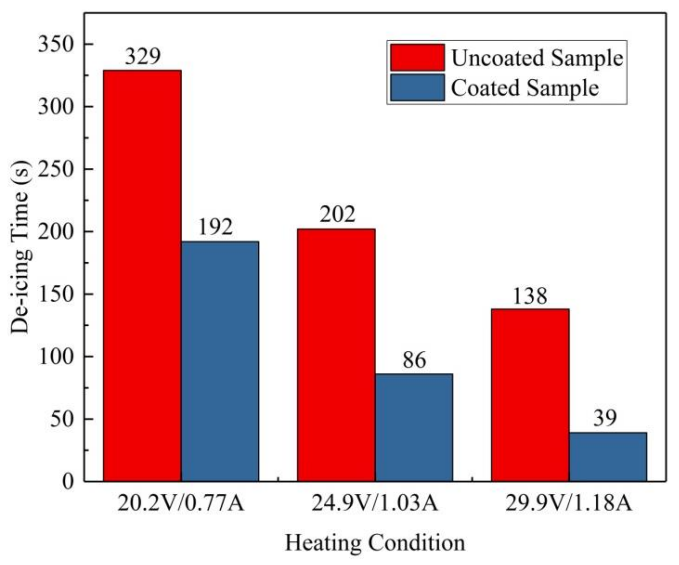

Figure 5. Deicing time under different heating conditions.

According to the energy conservation equation, the equal thickness icing needs equal energy for deicing in the same temperature environment $\left(Q=\frac{U^{2}}{R} t\right)$. Nevertheless, excluding the influence of the equivalent centrifugal force, the deicing time $t$ and the heating voltage $U$ do not satisfy the energy conservation equation. Excluding the factor of experimental error, the results can be explained as follows: The adhesion force between the icing and anti-/deicing component decreased in the heating condition, but formed no water film. The centrifugal force exerted on the icing increases the shear force between the contact surfaces of the anti-/deicing and icing components, which thereby accelerated the shedding of ice. Therefore, with the action of centrifugal force, the graphene coating can accelerate ice shedding from the anti-/deicing component, shorten the deicing time, and improve deicing efficiency.

\subsection{Spraying Process}

The spraying process influences the anti-/deicing efficiency of graphene coating noticeably. Given the single-layer nanostructure and the anisotropic heat-transfer characteristics of graphene, the spraying process of graphene should be further studied. In this paper, the influence of coating thickness and spraying pressure are studied.

Test samples with different coating thicknesses of graphene were prepared. The effects of coating thickness on the heat transfer and anti-/deicing performance of graphene were analyzed by measuring the thickness and surface temperature in the same refrigeration environment and heating conditions as the anti-/deicing components. The thickness of graphene was measured using the SEM image, and the temperature was measured with a thermocouple. The changes in heat transfer characteristics of the graphene coating were analyzed by recording the surface temperature variation curve, as shown in Figure 6.
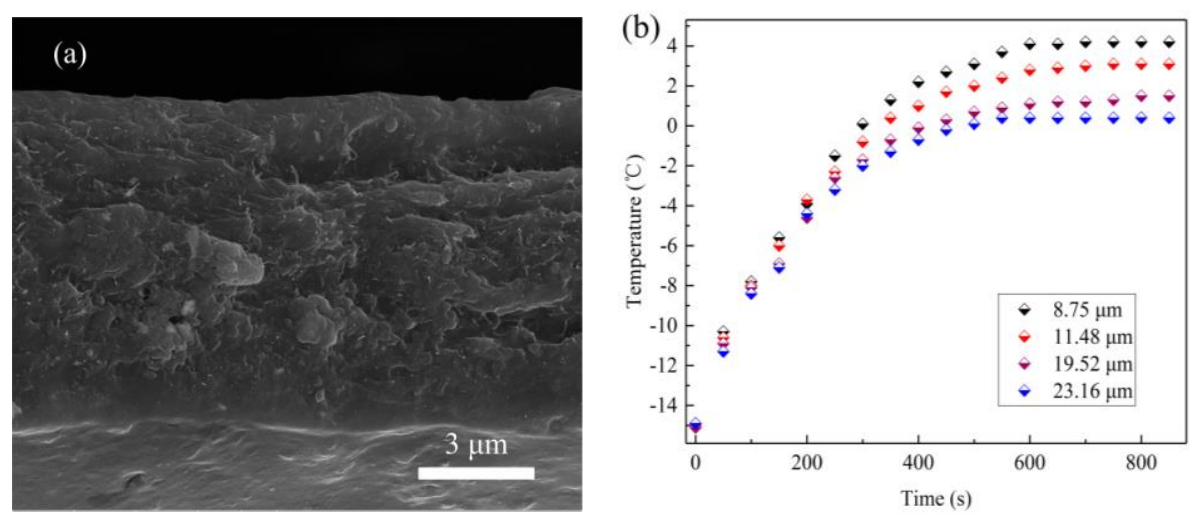

Figure 6. (a) SEM image and (b) temperature profile of the graphene coating with different thicknesses. 
Figure 6 illustrates that with increased coating thickness, the heat-transfer performance of graphene decreased. Under the same heating conditions as the anti-/deicing component, the surface temperature of graphene coatings decreased, which indicated that the ability of the graphene coating to prevent anti-icing and deicing was reduced. The experimental results can be explained by the following empirical formula of graphene thermal conductivity given by Berger [42] and Guo [43]:

$$
\lambda=\frac{X_{\mathrm{g}} L}{2 h d} \frac{\delta_{\mathrm{P}}}{\delta_{\mathrm{f}}}
$$

where $h$ is the thickness of single-layer graphene, $X_{\mathrm{g}}$ is the temperature coefficient, $L$ denotes the distance between the middle part of the single-layer graphene and the film, $d$ is the width of the single-layer graphene, $\delta_{\mathrm{f}}$ is the change in $\mathrm{G}$ peak displacement, and $\delta_{\mathrm{P}}$ is the change in thermal power. According to the empirical formula, the increase in graphene coating thickness results in decreased thermal conductivity, which indicates that the anti-/deicing ability of the graphene coating decreased. The heat-conduction mechanism shows that with the increase in graphene coating thickness, the arrangement of graphene atoms is more cluttered, which increases the resistance to thermal phonon propagation. Thus, the decrease in phonon propagation speed greatly affected the thermal conductivity of the graphene.

The effect of spraying pressure on the thermal conductivity of the graphene coating during the spray process was studied. Under the same experimental conditions, the graphene coating test samples were prepared using different spraying pressures. The surface temperature profiles of graphene coating with time were measured, and are shown in Figure 7. The influence of the spraying pressure of graphene on the heat-transfer performance was also analyzed.

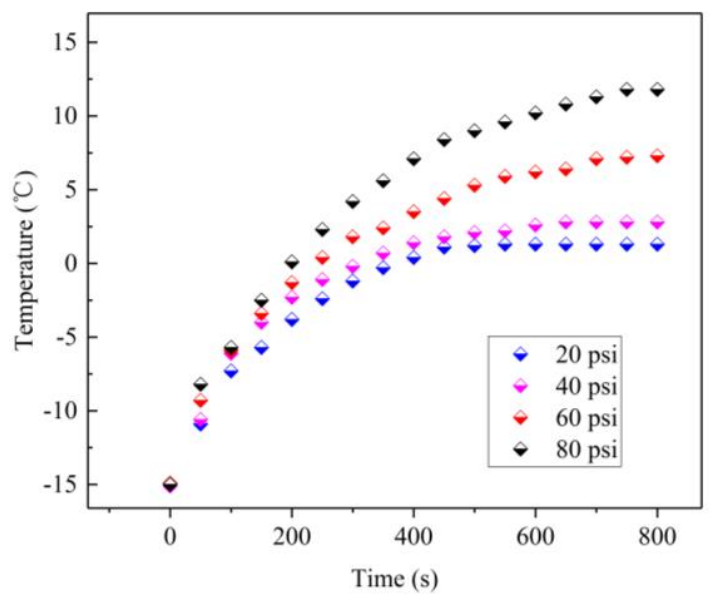

Figure 7. Temperature profile of graphene coating using different spaying pressures.

Figure 7 illustrates that the thermal conductivity of the graphene coating was gradually improved with increased spraying pressure. Under the same heating conditions as the anti-/deicing component, the surface temperature of the graphene coating prepared using high spraying pressure was evidently higher than that prepared using low spraying pressure. Therefore, spraying pressure influenced the graphene coating thermal conductivity. With increased spraying pressure, the graphene content in the unit spraying area also increased. Thus, the thermal conductivity of the graphene coating was increased, and the anti-/deicing capability of the anti-/deicing component was improved. By increasing the spray pressure, the graphene coating can be applied thinner, which leads to higher thermal conductivity. Therefore, by increasing spraying pressure, the graphene coating has better thermal conductivity. 


\section{Conclusions}

This work demonstrated the anti-/deicing efficiency of graphene coating by using its heat-transfer characteristics to improve the anti-/deicing component. The thermal conductivity of the graphene coating was applied to improve the heat-transfer performance of the anti-/deicing composite components. The effect of the graphene coating on the anti-icing and deicing efficiency of anti-/deicing component was also studied. The experimental data showed that with the action of centrifugal force, the graphene coating can enhance the heat transfer of the anti-/deicing component, effectively improve the anti-/deicing capacity, and reduce the energy consumption of the helicopter.

A reduced thickness of graphene coating can improve its thermal conductivity, thereby enhancing the anti-/deicing efficiency of the helicopter rotor. Graphene coating exhibited a good heat-transfer performance when using a high spraying pressure. The present results provide a reference for an application of the thermal conductivity of graphene coating.

By applying the graphene coating to improve the heat-transfer performance of the anti-/deicing component, the deicing efficiency of the anti-deicing component was greatly improved. Compared with the existing anti-/deicing method, maximum efficiency can be increased by $70 \%$. The conclusion of this study makes a significant contribution to the development of future helicopter rotor anti-/deicing technology.

Acknowledgments: This work was supported by Defense Industrial Technology Development Program (No. A0520110009), State Key Laboratory of Virtual Reality Technology Independent Subject (No. BUAA-VR-16ZZ-07).

Author Contributions: Long Chen and Yidu Zhang conceived and designed the experiments; Long Chen performed the experiments; Long Chen and Qiong Wu analyzed the data; Qiong Wu contributed analysis tools; Long Chen wrote the paper.

Conflicts of Interest: The authors declare no conflict of interest.

\section{Nomenclature}

d Width of the single-layer graphene, $\mathrm{mm}$

$L \quad$ Distance between the middle part of the single-layer graphene and the fins, $\mathrm{mm}$

$m_{\text {ice }} \quad$ Mass of the icing, $\mathrm{kg}$

$r \quad$ Radius of gyration, $\mathrm{m}$

$R \quad$ Resistance value, $\Omega$

$t \quad$ Heating time, $\mathrm{s}$

$Q \quad$ Joule heat energy, J

$U \quad$ Heating voltage, $\mathrm{V}$

$v \quad$ Rotating linear velocity of the icing, $\mathrm{m} / \mathrm{s}$

$X_{\mathrm{g}} \quad$ Temperature coefficient

$\lambda$ Thermal conductivity, $\mathrm{W} /\left(\mathrm{m} \cdot{ }^{\circ} \mathrm{C}\right)$

$\delta_{\mathrm{f}} \quad$ Change of $\mathrm{G}$ peak displacement

$\delta_{\mathrm{P}} \quad$ Change of thermal power

\section{References}

1. Coffman, H.J. Helicopter rotor icing protection methods. J. Am. Helicopter Soc. 1987, 32, 34-39. [CrossRef]

2. Zhang, X.; Wu, X.; Min, J. Aircraft icing model considering both rime ice property variability and runback water effect. Int. J. Heat Mass Transf. 2017, 104, 510-516. [CrossRef]

3. Zhao, G.Q.; Zhao, Q.J.; Chen, X. New 3-D ice accretion method of hovering rotor including effects of centrifugal force. Aerosp. Sci. Technol. 2016, 48, 122-130. [CrossRef]

4. Lee, J.S.; Yoon, J.C.; Jang, J.H. A route towards superhydrophobic graphene surfaces: Surface-treated reduced graphene oxide spheres. J. Mater. Chem. A 2013, 1, 7312-7315. [CrossRef]

5. Zhu, X.; Zhang, Z.; Ge, B. Fabrication of a superhydrophobic carbon nanotube coating with good reusability and easy repair ability. Colloids Surface A 2014, 444, 252-256. [CrossRef] 
6. Farhadi, S.; Farzaneh, M.; Kulinich, S.A. Anti-icing performance of superhydrophobic surfaces. Appl. Surf. Sci. 2011, 257, 6264-6269. [CrossRef]

7. Wang, Z.J.; Kwon, D.J.; Devries, K.L.; Park, J.M. Frost formation and anti-icing performance of a hydrophobic coating on aluminum. Exp. Therm. Fluid Sci. 2015, 60, 132-137. [CrossRef]

8. Moaven, K.; Rad, M.; Taeibi-Rahni, M. Experimental investigation of viscous drag reduction of superhydrophobic nano-coating in laminar and turbulent flows. Exp. Therm. Fluid Sci. 2013, 51, $239-243$. [CrossRef]

9. Liu, Q.; Yang, Y.; Huang, M.; Zhou, Y.X.; Liu, Y.Y.; Liang, X.D. Durability of a lubricant-infused electrospray silicon rubber surface as an anti-icing coating. Appl. Surf. Sci. 2015, 346, 68-76. [CrossRef]

10. Choi, B.G.; Park, H.S. Superhydrophobic graphene/nafion nanohybrid films with hierarchical roughness. J. Phys. Chem. C 2012, 116, 3207-3211. [CrossRef]

11. Buschhorn, S.T.; Kessler, S.S.; Lachmann, N.; Gavin, J.; Wardle, B.L.; Thomas, G. Electrothermal icing protection of aerosurfaces using conductive polymer nanocomposites. In Proceedings of the 54th AIAA Structures, Structural Dynamics, and Materials (SDM) Conference, Boston, MA, USA, 2013; pp. 1-8.

12. Nagappan, S.; Park, S.S.; Ha, C.S. Recent advances in superhydrophobic nanomaterials and nanoscale systems. J. Nanosci. Nanotechnol. 2014, 14, 1441-1443. [CrossRef] [PubMed]

13. Bu, X.Q.; Lin, G.P.; Yu, J.; Song, X. Numerical simulation of an airfoil electrothermal anti-icing system. Proc. Inst. Mech. Eng. Part G J. Aerosp. Eng. 2013, 227, 1608-1622. [CrossRef]

14. Pourbagian, M.; Habashi, W.G. Surrogate-based optimization of electrothermal wing anti-icing systems. J. Aircr. 2013, 50, 1555-1563. [CrossRef]

15. Ding, L.; Chang, S.; Leng, M. Transient characteristics of an electrothermal anti-icing process based on the improved messinger model. In Proceedings of the 2016 IEEE International Conference on Aircraft Utility Systems (AUS), Beijing, China, 10-12 October 2016; pp. 233-238.

16. Thomas, S.K.; Cassoni, R.P.; Macarthur, C.D. Aircraft anti-icing and de-icing techniques and modeling. J. Aircr. 2012, 33, 841-854. [CrossRef]

17. Yin, C.B.; Zhang, Z.D.; Wang, Z.J.; Guo, H. Numerical simulation and experimental validation of ultrasonic de-icing system for wind turbine blade. Appl. Acoust. 2016, 114, 19-26. [CrossRef]

18. Sandorff, P.E. Beam balancer electro-impulse deicing systems. U.S. Patent 4,501,398, 26 February 1985.

19. Bernhart, W.D.; Schrag, R.L. Electroimpulse deicing-Electrodynamic solution by discrete elements. J. Aircr. 2012, 26, 547-553. [CrossRef]

20. Tan, Y.W.; Zhang, Y.; Bolotin, K.; Zhao, Y. Measurement of scattering rate and minimum conductivity in graphene. Phys. Rev. Lett. 2007, 99, 246803. [CrossRef] [PubMed]

21. Kim, H.; Miura, Y.; Macosko, C.W. Graphene/polyurethane nanocomposites for improved gas barrier and electrical conductivity. Chem. Mater. 2010, 22, 3441-3450. [CrossRef]

22. Esfahani, M.R.; Languri, E.M. Exergy analysis of a shell-and-tube heat exchanger using graphene oxide nanofluids. Exp. Therm. Fluid Sci. 2016, 83, 100-106. [CrossRef]

23. Tian, L.M.; Wang, Y.J.; Li, Z.Y.; Mei, H.R.; Shang, Y.G. The thermal conductivity-dependant drag reduction mechanism of water droplets controlled by graphene/silicone rubber composites. Exp. Therm. Fluid Sci. 2017, 85, 363-369. [CrossRef]

24. Jang, S.; Jang, H.; Lee, Y.; Sub, D.; Baik, S.; Hong, B.H.; Ahn, J.H. Flexible, transparent single-walled carbon nanotube transistors with graphene electrodes. Nanotechnology 2010, 21, 425201. [CrossRef] [PubMed]

25. Kang, J.; Kim, H.; Kim, K.S.; Lee, S.K.; Bae, S.; Ahn, J.H.; Kim, Y.J.; Choi, J.B.; Hong, B.H. High-performance graphene-based transparent flexible heaters. Nano Lett. 2011, 11, 5154-5158. [CrossRef] [PubMed]

26. Yu, D.; Dai, L. Self-assembled graphene/carbon nanotube hybrid films for super capacitors. J. Phys. Chem. Lett. 2015, 1, 467-470. [CrossRef]

27. Zhu, Y.; Murali, S.; Stoller, M.D.; Ganesh, K.J.; Cai, W.W.; Ferreira, P.J.; Pirkle, A.; Wallace, R.M.; Cychosz, K.A.; Thommes, M.; et al. Carbon-based supercapacitors produced by activation of graphene. Science 2011, 332, 1537-1541. [CrossRef] [PubMed]

28. Kamyshny, A.; Magdassi, S. Conductive nanomaterials for printed electronics. Small 2014, 10, 3515-3535. [CrossRef] [PubMed]

29. Secor, E.B.; Ahn, B.Y.; Gao, T.Z.; Lewis, J.A.; Hersam, M.C. Rapid and versatile photonic annealing of graphene inks for flexible printed electronics. Adv. Mater. 2015, 27, 6683-6688. [CrossRef] [PubMed]

30. Lu, F.; Botte, G.G. Ammonia generation via a graphene-coated nickel catalyst. Coatings 2017, 7, 72. 
31. Chen, J.Z.; Chiu, Y.F.; Chen, C.W.; Hsu, C.C.; Cheng, I.C. Atmospheric-pressure plasma jet processed Pt-decorated reduced graphene oxides for counter-electrodes of dye-sensitized solar cells. Coatings 2016, 6,44 .

32. Volman, V.; Zhu, Y.; Raji, A.R.; Genorio, B.; Lu, W.; Xiang, C.; Kittrell, C.; Tour, J.M. Radio-frequencytransparent, electrically conductive graphene nanoribbon thin films as deicing heating layers. ACS Appl. Mater. Interfaces 2014, 6, 298-304. [CrossRef] [PubMed]

33. Raji, A.R.; Varadhachary, T.; Nan, K.; Wang, T.; Lin, J.; Ji, Y.; Genorio, B.; Zhu, Y.; Kittrell, C.; Tour, J.M. Composites of graphene nanoribbon stacks and epoxy for joule heating and deicing of surfaces. ACS Appl. Mater. Interfaces 2016, 8, 3551-3556. [CrossRef] [PubMed]

34. Wang, T.; Zheng, Y.; Raji, A.R.; Li, Y.; Sikkema, W.K.; Tour, J.M. Passive anti-icing and active deicing films. ACS Appl. Mater. Interfaces 2016, 8, 14169-14173. [CrossRef] [PubMed]

35. Ellahi, R.; Shivanian, E.; Abbasbandy, S.; Hayat, T. Numerical study of magnetohydrodynamics generalized couette flow of eyring-powell fluid with heat transfer and slip condition. Int. J. Numer. Methods Heat 2016, 26, 1433-1445. [CrossRef]

36. Rashidi, S.; Esfahani, J.A.; Ellahi, R. Convective heat transfer and particle motion in an obstructed duct with two side by side obstacles by means of DPM model. Appl. Sci. 2017, 7, 431. [CrossRef]

37. Shirvan, K.M.; Mamourian, M.; Mirzakhanlari, S.; Ellahi, R. Two phase simulation and sensitivity analysis of effective parameters on combined heat transfer and pressure drop in a solar heat exchanger filled with nanofluid by RSM. J. Mol. Liq. 2016, 220, 888-901. [CrossRef]

38. Shirvan, K.M.; Ellahi, R.; Mirzakhanlari, S.; Mamourian, M. Enhancement of heat transfer and heat exchanger effectiveness in a double pipe heat exchanger filled with porous media: Numerical simulation and sensitivity analysis of turbulent fluid flow. Appl. Therm. Eng. 2016, 109, 761-774. [CrossRef]

39. Shirvan, K.M.; Mamourian, M.; Mirzakhanlari, S.; Ellahi, R.; Vafai, K. Numerical investigation and sensitivity analysis of effective parameters on combined heat transfer performance in a porous solar cavity receiver by response surface methodology. Int. J. Heat Mass Transf. 2017, 105, 811-825. [CrossRef]

40. Bhatti, M.M.; Zeeshan, A.; Ellahi, R. Electromagnetohydrodynamic (EMHD) peristaltic flow of solid particles in a third-grade fluid with heat transfer. Mech. Ind. 2017, 18, 314. [CrossRef]

41. Civil Aviation Administration of China; Miller, F.P., Vandome, A.F., McBrewster, J., Eds.; Alphascript Publishing: Beau Bassin, Mauritius, 2010.

42. Berger, C. Electronic confinement and coherence in patterned epitaxial graphene. Science 2006, 312, 1191-1196. [CrossRef] [PubMed]

43. Guo, Z.; Zhang, D.; Gong, X.G. Thermal conductivity of graphene nanoribbons. Appl. Phys. Lett. 2009, 95, 163103. [CrossRef]

(C) 2017 by the authors. Licensee MDPI, Basel, Switzerland. This article is an open access article distributed under the terms and conditions of the Creative Commons Attribution (CC BY) license (http://creativecommons.org/licenses/by/4.0/). 